\title{
Validação Fatorial do "Spanish Burnout Inventory" em Policiais Portugueses
}

\author{
Factorial Validity of the "Spanish Burnout Inventory" \\ among Portuguese Police Officers
}

\author{
Hugo Figueiredo-Ferraz ${ }^{*},{ }$, Pedro R. Gil-Monte ${ }^{a}$, Cristina Queirós $^{b} \&$ Fernando Passos $^{c}$ \\ ${ }^{a}$ Universitat de València, València, Provincia de València, España, \\ ${ }^{b}$ Universidade do Porto, Porto, Distrito de Porto, Portugal \\ $\&^{c}$ Gabinete de Psicologia da Policía de Segurança Pública, Lisboa, Distrito de Lisboa, Portugal
}

\begin{abstract}
Resumo
O objetivo deste estudo foi explorar a estrutura fatorial e a consistência interna da adaptação ao português do "Spanish Burnout Inventory" (SBI) numa amostra composta por 245 policiais de diversos esquadrões da região de Lisboa. Realizou-se uma análise fatorial exploratória (AFE), de onde foram extraídos 4 fatores com autovalor $>1$, que reproduziram a distribuição dos itens da versão original: no primeiro fator ficaram agrupados os itens da subescala Entusiasmo pelo Trabalho, no segundo os itens da subescala Culpa, no terceiro os itens da subescala Indolência, sendo que os itens da subescala Desgaste Psíquico se distribuíram pelo quarto fator. Todas as subescalas do SBI alcançaram valores alfa de Cronbach superiores a 0,70. Os resultados sugerem que a versão portuguesa do SBI é um instrumento valido e fiável para avaliar a síndrome do burnout em policiais portugueses.

Palavras-chave: Esgotamento profissional, policiais, SBI, estresse ocupacional, análise fatorial.
\end{abstract}

\begin{abstract}
This study was designed to assess the factor structure and the internal consistency of the Portuguese adaptation of the "Spanish Burnout Inventory" (SBI) in a sample of 245 Portuguese police officers working in Lisbon. Using an Exploratory Factor Analysis (EFA), 4 factors were extracted with eigenvalue $>1$, reproducing the original model: the first factor was constituted by the items of "enthusiasm toward the job", the second factor was constituted by the items of "guilt", the third factor was constituted by the items of "indolence", and the fourth factor was constituted by the items of "psychological exhaustion". Internal consistency values for all subscales showed values of Cronbach's alpha higher than .70. The results suggest that the Portuguese adaptation of the SBI is an adequate instrument to assess burnout in Portuguese police officers.

Keywords: Burnout, police officers, SBI, job stress, factorial analysis.
\end{abstract}

Os elementos das forças policiais confrontam-se com uma multiplicidade de acontecimentos, a maioria dos quais exigindo resolução imediata e o confronto com a imprevisibilidade e incerteza (Ransley \& Mazerolle, 2009). Estão, por isso, vulneráveis ao estresse e ao burnout (Ranta \& Sud, 2008). O policiamento tem sido referido como uma atividade estressante e em sucessivos estudos comparativos sobre estresse ocupacional ser policial é considerada a segunda atividade mais estressante (Johnson et al., 2005). Desde o interesse científico manifestado na década de sessenta sobre a atividade policial, inúmeros estudos foram efetuados sobre o estresse e burnout sentidos pelos

\footnotetext{
* Endereço para correspondência: Departamento de Psicología Social, Unidad de Investigación Psicosocial de la Conducta Organizacional, Universitat de València, Av. Blasco Ibáñez, 21, València, España 46010. E-mail: hufidema@postal.uv.es
}

policiais durante o desempenho das suas funções, sejam estas de tipo administrativo, patrulhamento ou investigação criminal (Akinola \& Mendes, 2012; Schaible \& Gecas, 2010; Violanti et al., 2009), existindo a preocupação de que o estresse nos policiais pode levar estes ao suicídio devido ao fácil acesso à arma (Hackett \& Violanti, 2003).

A comunidade científica tem-se interessado de forma crescente pelo tema, e o estresse e burnout nos policiais têm sido alvo de estudos recentes em vários países da América, como por exemplo, USA (Gachter, Savage, \& Torgler, 2011), Brasil (Coleta \& Coleta, 2008) e na Europa: Portugal (Afonso \& Gomes, 2009), Espanha (Durán, Montalbán, \& Stangeland, 2006), Holanda (van Gelderen, Bakker, Konijn, \& Demerouti, 2011).

De forma consistente, os inúmeros estudos com policiais enfatizam que o desempenho das atividades ligadas ao policiamento pode provocar, para além do estresse e burnout, doenças físicas, depressão, agressividade e até 
o suicídio. Dos principais estressores referidos comuns a diferentes países, podem-se destacar por exemplo ter de matar alguém durante o policiamento, excesso de burocracia e falta de suporte das chefias (Ravelo, Garcia, \& Dorta, 2009), supressão de emoções e conflito entre emoções sentidas e expressas (van Gelderen et al., 2011), desequilíbrio entre recursos e exigências (Euwema, Kop, \& Bakker, 2004), perigo e rotina ou incertezas durante o policiamento no terreno (Pancheri et al., 2002), baixo salário e hostilidade dos cidadãos (Pines \& Keinan, 2005, 2006) e falta de preparação e imagem pública da Polícia (Agolla, 2009).

Desde que Herbert Freudenberger (1974) definiu o burnout como um estado de esgotamento físico e mental, cuja causa está intimamente ligada à vida profissional, muitos têm sido os estudos sobre este fenómeno. Atualmente, a definição aceite mais frequentemente pela comunidade científica é a de Maslach, Schaufeli e Leiter (2001), que definem burnout e as suas três dimensões: exaustão emocional, que se caracteriza por uma falta ou carência de energia, entusiasmo e sentimento de esgotamento de recursos; despersonalização, que se caracteriza por tratar os clientes, colegas e a organização como objetos; diminuição da realização pessoal no trabalho, que é caracterizada por uma tendência do trabalhador a se autoavaliar de forma negativa. As pessoas sentem-se infelizes e insatisfeitas com o seu desenvolvimento profissional e estes sintomas são avaliados mediante o Maslach Burnout Inventory (MBI; Maslach \& Jackson, 1986). Este questionário é o mais utilizado para a avaliação psicométrica do burnout. O MBI avalia os índices da síndrome de acordo com os resultados obtidos em cada dimensão, sendo que altas pontuações em exaustão emocional e despersonalização, e baixas pontuações em realização pessoal indicam um alto nível de burnout.

O MBI é por excelência o teste mais utilizado para a medição do burnout, independentemente das características ocupacionais de amostra e da sua origem. Segundo a última atualização do manual (Maslach, Jackson, \& Leiter, 1996) existem três versões do MBI: MBI-Human Services Survey (MBI-HSS), dirigido a profissionais de saúde, que é a versão clássica do MBI (Maslach \& Jackson, 1986), MBI-General Survey (MBI-GS) e o MBI-Educators Survey (MBI-ES; Maslach, Jackson, \& Schwab, 1986).

Na versão original americana, a consistência interna das três dimensões do questionário é satisfatória, pois apresenta um alfa de Cronbach que vai de 0,71 até 0,90 enquanto os coeficientes de teste e re-teste vão de 0,60 a 0,80 em períodos de até um mês (Maslach \& Jackson, 1981). Diferentes estudos de caráter exploratório reproduziram uma estrutura de três fatores, similar à do manual para as diferentes versões do MBI já referidas, quer por rotação ortogonal (Mota-Cardoso, Araújo, Carreira Ramos, Gonçalves, \& Ramos, 2002; Soderfeldt, Soderfeldt, Warg, \& Ohlson, 1996), quer por rotação oblíqua (Abu-Hilal, 1995; Gold, Bachelor, \& Michael, 1989). Contudo, alguns estudos de validação do MBI têm apresentado também diferentes distribuições fatoriais, normalmente variando de 3 a 5 fatores (Carlotto \& Gonçalves, 2004). Alguns estudos apresentam apenas dois fatores (Kalliath, O'Driscoll, Gillespie, \& Bluedorn, 2000), outros quatro (Iwanicki \& Schwab, 1981) e outros, até seis fatores (Pedrabissi, Santinello, \& Vialetto, 1994).

Outro problema psicométrico do MBI está relacionado com os valores do alfa de Cronbach relativamente baixos que, com certa frequência, se obtêm para a subescala de despersonalização, existindo estudos onde o alfa de Cronbach varia entre 0,42 e 0,64 (Bhana \& Haffejee, 1996; Carlotto \& Gonçalves, 2004; Peeters \& Rutte, 2005). Algumas dessas limitações foram encontradas em estudos realizados em Portugal. Por exemplo, para a subescala de despersonalização do questionário $\mathrm{MBI}$, foi encontrado num estudo com enfermeiros um valor alfa de Cronbach igual a 0,52 (Sá \& Fleming, 2008), e mais recentemente, num estudo realizado com enfermeiros e professores um valor alfa de Cronbach igual a 0,65 (Figueiredo-Ferraz, Gil-Monte, \& Grau-Alberola, 2013). Na mesma linha encontrou-se que a subescala de cinismo (MBI-GS) num estudo feito em Portugal com a Guarda Nacional Republicana obteve um valor alfa de Cronbach igual a 0,64 (Afonso \& Gomes, 2009). Outra limitação, e também relacionada com a subescala de despersonalização foi a encontrada num estudo com professores, onde a escala se dividiu em dois fatores (Marques-Pinto, Lima, \& Lopes da Silva, 2005).

A partir da criação do MBI, ao longo dos anos, muitos foram os instrumentos desenvolvidos por vários autores, com o intuito de melhorar as suas deficiências, como por exemplo, o Tedium Measure (Burnout Measure; Pines, Aronson, \& Kafry, 1981), Holland Burnout Assessment Survey (Holland \& Michael, 1993) e mais recentemente o Oldenburg Burnout Inventory (Halbesleben \& Demerouti, 2005), The Copenhagen Burnout Inventory (Kristensen, Borrritz, Villadsen, \& Christensen, 2005) e, o Spanish Burnout Inventory (SBI) ${ }^{1}$ (Gil-Monte, Figueiredo-Ferraz, \& Valdez-Bonilla, 2013).

O modelo teórico do SBI foi desenvolvido por Gil-Monte (2005) e considera que o burnout é uma resposta característica ao estresse laboral crônico dos profissionais que trabalham para pessoas. Neste modelo a deterioração cognitiva (baixas pontuações na dimensão Entusiasmo pelo trabalho) e afetiva (altas pontuações na dimensão Desgaste Psíquico) aparecem, num primeiro momento, como resposta às fontes de estresse laboral crônico, para em seguida os indivíduos desenvolverem atitudes negativas em relação às pessoas que atendem no seu trabalho (alto níveis de Indolência). O aparecimento dos sentimentos de culpa é posterior a estes sintomas mas não é apresentado por todos os indivíduos. Deste modo, é possível distinguir dois perfis no processo do burnout: num primeiro perfil, os indivíduos apresentam baixo entusiasmo pelo trabalho e altos níveis de desgaste psíquico e indolência, mas não

\footnotetext{
${ }^{1} \mathrm{O}$ nome em espanhol do SBI é "Cuestionario para la Evaluación del Síndrome de Quemarse por el Trabajo" (CESQT).
} 
Figueiredo-Ferraz, H., Gil-Monte, P. R., Queirós, C. \& Passos, F. (2014). Validação Factorial do "Spanish Burnout Inventory" em Policiais Portugueses.

apresentam sentimentos de culpa; num segundo perfil, mais grave, os indivíduos apresentam também sentimentos de culpa.

A utilização deste instrumento surge na sequência das insuficiências que apresentam outros instrumentos para a avaliação da síndrome do burnout na sua adaptação ao castelhano e ao português (Marques-Pinto et al., 2005). Os instrumentos psicométricos mais utilizados na atualidade (e.g., Maslach Burnout Inventory, Burnout Measure) demonstram rigor científico e validade psicométrica, mas apresentam algumas insuficiências de conteúdo e psicométricas que se acentuam quando se adaptam a diferentes idiomas, sendo em alguns estudos questionável a sua fiabilidade e a sua validade.

Com o objetivo de criar um instrumento de avaliação da síndrome de burnout que ultrapasse as críticas anteriores, Gil-Monte (2005) elaborou o SBI, questionário formado por 20 itens distribuídos por quatro dimensões: (a) Entusiasmo pelo trabalho (5 itens): define-se como o desejo do indivíduo alcançar as metas laborais que considera serem uma fonte de prazer pessoal. O indivíduo percebe o seu trabalho como atrativo e alcançar as metas profissionais é uma fonte de realização pessoal. Os itens que compõem esta dimensão estão formulados de maneira positiva, e baixas pontuações nesta dimensão indicam altos níveis de burnout. (b) Desgaste psíquico (4 itens): aparecimento de esgotamento emocional e físico devido ao fato de no trabalho o indivíduo ter de lidar, várias vezes por dia, com pessoas que apresentam ou causam problemas. (c) Indolência (6 itens): presença de atitudes negativas de indiferença e cinismo para com os clientes da organização. Os indivíduos que pontuam alto nesta dimensão mostram insensibilidade e não se comovem perante os problemas das pessoas para quem trabalham. (d) Culpa (5 itens): para a inclusão neste questionário, tomaram-se como referência estudos em que se considera que os sentimentos de culpa são um sintoma característico de indivíduos que desenvolvem burnout.

Tendo em conta a estrutura fatorial do SBI, anteriores estudos realizados com análise fatorial exploratória (AFE) mostraram uma estrutura de quatro fatores semelhante à do modelo: Entusiasmo pelo trabalho, Desgaste psíquico, Indolência e Culpa (Olivares \& Gil-Monte, 2007). No mesmo sentido, o SBI obteve valores adequados de consistência interna, validade fatorial, e validade concorrente com o MBI em diferentes estudos (Gil-Monte, Carretero, Roldán, \& Núñez-Román 2005) realizados com análise fatorial confirmatória (AFC; Figueiredo-Ferraz et al., 2013; Gil-Monte \& Olivares, 2011). Com base nestes resultados, pode-se concluir que para avaliar o burnout, o SBI é um instrumento válido e fiável. Os resultados confirmaram a estrutura hipotetizada de quatro fatores, o que contribui para a validação psicométrica do questionário. No entanto, embora estudos anteriores, com enfermeiros espanhóis (Gil-Monte, 2008), professores e enfermeiros portugueses (Figueiredo-Ferraz et al., 2013), profissionais que trabalham com pessoas que possuem deficiência intelectual (Gil-Monte \& Figueiredo-Ferraz, 2013) tenham já confirmado a estrutura de quatro fatores do SBI, estes foram realizados em profissionais que trabalham em sectores profissionais diferentes dos policiais. Dessa forma, este grupo profissional, que tem condições de trabalho e características sociodemográficas diferentes (p.e., mais homens que mulheres), poderia levar a que se encontrem diferenças nas propriedades psicométricas e na validade do SBI. Os policiais portugueses são na sua maioria homens, e o genero é uma variável que explica diferenças significativas no burnout (Purvanova \& Muros, 2010). A maioria dos estudos anteriores que examinaram as propriedades psicométricas do SBI foram desenvolvidos com amostras em que a maioria dos participantes eram mulheres, por exemplo, em professores (Gil-Monte, Carlotto, \& Gonçalves, 2010; Gil-Monte, Unda, \& Sandoval, 2009; Mercado \& Gil-Monte, 2012: 77,5\%, 86,6\%, e 54\% das amostras eram mulheres, respetivamente), em profissionais que trabalham com pessoas com deficiência intelectual $(75,8 \%$ das mulheres; Gil-Monte et al., 2005) e funcionários administrativos que lidam com o público (75\% mulheres; Olivares \& Gil-Monte, 2007). A generalização da estrutura de quatro-fatores do SBI obtidos em anteriores estudos deve ser vista com cautela, porque os valores culturais do trabalho e as condições de trabalho, que são preditores de burnout (Mann-Feder \& Savicki, 2003) são variáveis influenciadas pelas diferenças culturais. Tendo em consideração as características da amostra deste estudo, esta é mais uma razão que fortalece a justificação para levar a cabo o mesmo.

O objetivo deste estudo é analisar a validade fatorial do "Spanish Burnout Inventory", e a consistência interna das suas subescalas em profissionais da Polícia portuguesa. Tomando como referência o objetivo de estudo, hipotetiza-se que a solução da AFE oferecerá quatro fatores similares aos obtidos para a versão espanhola.

\section{Metodologia}

\section{Amostra}

A amostra deste estudo é constituída por 245 elementos da Polícia de Segurança Pública (PSP), a desempenharem funções em batalhões da área metropolitana de Lisboa. $\mathrm{Na}$ amostra, 213 sujeitos pertencem ao sexo masculino $(86,9 \%)$ e 26 ao sexo feminino (10,6\%), existindo ainda 6 sujeitos $(2,4 \%)$ que não responderam a esta questão. Sendo elementos da PSP, todos os sujeitos têm contrato efetivo. A Média de idade da amostra foi de 33,57 (DP $=7,51$, $\max .=54$, min. $=22)$. Em relação ao tempo de profissão, a Média obtida foi de 10,28 $(D P=7,72$, max. $=$ 31 , min. $=1)$. Quanto ao tempo na organização, a Média foi de $10,25(D P=7,72, \max .=31, \min .=1)$. A taxa de resposta foi de $81,6 \%$.

\section{Instrumento}

Os dados foram coletados através do SBI na sua adaptação para português (Figueiredo-Ferraz et al., 2013; Gil-Monte et al., 2010). O questionário é constituído por 20 
itens distribuídos por quatro dimensões designadas como: Entusiasmo pelo trabalho (5 itens), Desgaste psíquico (4 itens), Indolência (6 itens) e Culpa (5 itens). Os itens são avaliados através de uma escala de frequência de 5 níveis onde o valor (0) representa "Nunca" e o valor (4) "Muito frequentemente". Baixas pontuações em Entusiasmo pelo trabalho e altas pontuações em Desgaste psíquico e Indolência representam altos níveis da síndrome do Burnout. No caso de sujeitos com o Perfil 2, estas altas pontuações são ainda acompanhadas de alta pontuação na dimensão Culpa.

\section{Procedimento}

Os questionários foram coletados em 2011, no Comando Metropolitano de Lisboa, Portugal, após autorização formal da Direção Nacional da Polícia de Segurança Pública e com a colaboração do Gabinete de Psicologia. Foram contactados os comandantes de vários batalhões de Lisboa e aos que aceitaram colaborar foram entregues 300 questionários, dos quais foram devolvidos preenchidos 245. Para solicitar voluntários, os comandantes informaram seus subalternos sobre uma pesquisa da Universidade do Porto para adaptação para o português da versão espanhola de um questionário sobre estresse nas forças policiais. Foi entregue aos elementos policiais que aceitaram colaborar o SBI numa versão em papel, que preencheram de forma anônima e colocaram numa caixa fechada, não sendo possível à instituição conhecer os resultados nem existindo contato direto com os pesquisadores. Os questionários preenchidos foram enviados para a Universidade do Porto e os dados introduzidos numa matriz do SPSS versão 20, mantendo o anonimato e confidencialidade dos resultados.

\section{Resultados}

Antes de realizar a AFE do SBI, foi feita uma análise de itens para comprovar a sua qualidade psicométrica. Todos os itens apresentam valores de assimetria e curtose no intervalo +/- 1, com exceção do item 13 e do item 16, que pertencem à escala de Culpa e apresentam um valor de assimetria e curtose superior a $+/-1$ (Tabela 1).

Analisando a contribuição dos itens para a consistência interna da escala à qual pertencem, foi constatado que todos os itens contribuem para a consistência interna da escala pois se o item é eliminado, o valor global do alfa de Cronbach da escala diminui. Exceto os itens 14 (que pertence à escala de Indolência) e 20 (que pertence à escala de Culpa) não contribuem para a consistência interna da escala, pois se são eliminados o valor global do alfa de Cronbach da escala aumenta. Da mesma forma, que no que diz respeito aos índices de homogeneidade corrigida, todos os itens variam de 0,41 a 0,79 , valores que ficam muito acima de 0,30 , o que nos assegura a fiabilidade dos itens em particular e das escalas em geral, sendo que os itens 14 e 20 obtiveram valores inferiores a 0,30 (Tabela 2).

Com relação às escalas do questionário, apenas a escala de Indolência apresenta um valor de curtose superior a +/-
1, enquanto as restantes apresentam na sua globalidade, valores de assimetria e curtose entre $+/-1$ (Tabela 2).

Tabela 1

Média, Desvio Padrão, Assimetria e Curtose para os Itens do $S B I(n=245)$

\begin{tabular}{|c|c|c|c|}
\hline & $M(D P)$ & Assimetria & Curtose \\
\hline \multicolumn{4}{|c|}{ Entusiasmo pelo trabalho } \\
\hline Item 1 & $2,68(0,92)$ & $-0,68$ & 0,63 \\
\hline Item 5 & $2,62(1,11)$ & $-0,40$ & $-0,64$ \\
\hline Item 10 & $2,46(1,03)$ & $-0,37$ & 0,29 \\
\hline Item 15 & $2,53(1,18)$ & $-0,56$ & $-0,52$ \\
\hline Item 19 & $2,22(1,14)$ & $-0,25$ & $-0,78$ \\
\hline \multicolumn{4}{|c|}{ Desgaste psíquico } \\
\hline Item 8 & $1,25(1,02)$ & 0,79 & 0,27 \\
\hline Item 12 & $1,37(1,09)$ & 0,59 & $-0,71$ \\
\hline Item 17 & $1,62(1,06)$ & $-0,35$ & $-0,35$ \\
\hline Item 18 & $1,80(1,25)$ & 0,21 & $-0,66$ \\
\hline \multicolumn{4}{|l|}{ Indolência } \\
\hline Item 2 & $1,45(0,96)$ & 0,28 & $-0,28$ \\
\hline Item 3 & $1,65(0,88)$ & 0,35 & 0,33 \\
\hline Item 6 & $1,16(0,92)$ & 0,61 & 0,37 \\
\hline Item 7 & $0,96(1,00)$ & 1,00 & 0,77 \\
\hline Item 11 & $1,31(1,00)$ & 0,56 & 0,07 \\
\hline Item 14 & $1,55(1,21)$ & 0,42 & $-0,80$ \\
\hline \multicolumn{4}{|l|}{ Culpa } \\
\hline Item 4 & $1,28(1,22)$ & 0,77 & $-0,40$ \\
\hline Item 9 & $0,97(0,99)$ & 0,93 & 0,21 \\
\hline Item 13 & $0,72(0,87)$ & 1,22 & 1,30 \\
\hline Item 16 & $0,83(0,85)$ & 1,26 & 2,18 \\
\hline Item 20 & $1,25(0,81)$ & 0,53 & 0,92 \\
\hline
\end{tabular}

Nota. O número do item corresponde à sua posição no questionário.

Consideraram-se ainda alguns critérios para avaliar a viabilidade da AFE: o determinante da matriz de correlações apresentou um valor de 0,000 , a prova de esfericidade de Bartlett foi significativa e o teste KMO de adequação da amostra alcançou um valor $>0,80$.

Como se observa na Tabela 3, utilizando uma solução de Componentes Principais e Rotação Varimax, obtiveram-se 4 fatores que explicam 59,25\% da variância 
Figueiredo-Ferraz, H., Gil-Monte, P. R., Queirós, C. \& Passos, F. (2014). Validação Factorial do “Spanish Burnout Inventory" em Policiais Portugueses.

Tabela 2

Homogeneidade Corrigida, Correlação Múltipla ao Quadrado, e Índice de Fiabilidade para os Itens do SBI por Escalas

\begin{tabular}{|c|c|c|c|}
\hline & $\begin{array}{l}\text { Homogeneidade } \\
\text { corrigida }\end{array}$ & $\begin{array}{l}\text { Correlação } \\
\text { múltipla ao } \\
\text { quadrado }\end{array}$ & $\begin{array}{l}\text { Alfa ao se } \\
\text { eliminar o } \\
\text { elemento }\end{array}$ \\
\hline \multicolumn{4}{|c|}{ Entusiasmo pelo trabalho } \\
\hline Item 1 & 0,68 & 0,49 & 0,88 \\
\hline Item 5 & 0,73 & 0,55 & 0,86 \\
\hline Item 10 & 0,70 & 0,51 & 0,87 \\
\hline Item 15 & 0,79 & 0,64 & 0,85 \\
\hline Item 19 & 0,76 & 0,59 & 0,86 \\
\hline \multicolumn{4}{|c|}{ Desgaste psíquico } \\
\hline Item 8 & 0,61 & 0,40 & 0,63 \\
\hline Item 12 & 0,49 & 0,31 & 0,70 \\
\hline Item 17 & 0,53 & 0,29 & 0,68 \\
\hline Item 18 & 0,49 & 0,27 & 0,70 \\
\hline \multicolumn{4}{|l|}{ Indolência } \\
\hline Item 2 & 0,55 & 0,32 & 0,66 \\
\hline Item 3 & 0,41 & 0,27 & 0,70 \\
\hline Item 6 & 0,53 & 0,33 & 0,66 \\
\hline Item 7 & 0,48 & 0,29 & 0,68 \\
\hline Item 11 & 0,55 & 0,31 & 0,65 \\
\hline Item 14 & 0,28 & 0,11 & 0,75 \\
\hline \multicolumn{4}{|l|}{ Culpa } \\
\hline Item 4 & 0,52 & 0,34 & 0,66 \\
\hline Item 9 & 0,65 & 0,45 & 0,60 \\
\hline Item 13 & 0,59 & 0,38 & 0,63 \\
\hline Item 16 & 0,54 & 0,34 & 0,65 \\
\hline Item 20 & 0,14 & 0,03 & 0,78 \\
\hline
\end{tabular}

total. Seguindo o critério de englobar cada item no fator onde a sua carga fatorial tenha sido superior a 0,40 , no Fator I (19,5\% da variância), ficaram agrupados os itens da escala Entusiasmo pelo trabalho (itens 1, 5, 10, 15, 19), e o item 18 que pertence à escala de Desgaste psíquico. No Fator II (14,2\% da variância), ficaram agrupados os itens $4,9,13$, e 16, que pertencem à escala de Culpa. No Fator III (13,8\% da variância), ficaram agrupados os itens que compõem a escala de Indolência (itens 2, 3, 6, 7, $11 \mathrm{e}$ 14), e o item 20 que pertence à escala de Culpa. Por fim, no Fator IV (11,7\% da variância), ficaram agrupados os itens da escala Desgaste psíquico (itens 8,12, 17 e 18), e o item 20 da escala de Culpa.

Considerando 0,40 como critério para englobar cada item no fator (Nunnally, 1978), todos os itens se agruparam no fator ao qual pertenciam por conteúdo, à exceção do item 20 que se agrupou em dois fatores, nenhum deles sendo o fator onde por conteúdo se deveria ter agrupado. Por sua vez, o item 18 obteve uma carga fatorial superior a 0,40 no fator 1 , mas foi muito superior no fator 4 , no qual se deveria ter agrupado. Por fim, o item 14 obteve uma carga fatorial de 0,39 , muito próxima de 0,40 .

$\mathrm{Na}$ Tabela 4 são apresentadas as estatísticas descritivas e correlações para as escalas do SBI. Todas as escalas do questionário obtiveram valores alfa de Crobach superiores a 0,70. Como era de esperar, as correlações entre Entusiasmo pelo trabalho e as outras escalas são negativas e significativas, enquanto as correlações entre as dimensões restantes são positivas e significativas.

\section{Discussão}

O objetivo deste trabalho era a validação do SBI para policiais portugueses. Perante os resultados obtidos pode-se afirmar que o SBI é um instrumento válido e fiável para a avaliar a síndrome do burnout, em profissionais da Polícia.

Os resultados apresentam uma estrutura fatorial muito similar à hipotetizada, isto é, foi obtido um fator que agrupa os itens que medem a Entusiasmo pelo trabalho, um fator que agrupa os itens que medem o Desgaste psíquico, um fator que agrupa os itens que medem Indolência e um fator que agrupa os itens que medem Culpa. Com base nestes resultados, podemos afirmar que os fatores 1, 2, 3 e 4 se diferenciam de forma clara. Apenas o item 20 apresenta cargas excessivamente baixas no fator no qual deveria se agrupar dado o seu conteúdo. Por outro lado, o item 14 que pertence a escala Indolência apresenta uma carga fatorial inferior a 0,40 no fator no qual deveria se agrupar, sendo no entanto esta a carga mais elevada desse item num fator. Nesse sentido, num estudo anterior, com uma amostra similar, que tinha como objetivo analisar a estrutura fatorial do SBI em guardas prisionais mexicanos, utilizando analise fatorial confirmatório (AFC), o item 14 foi o que obteve a relação item-fator mais baixa do estudo $(\lambda=0,31$; Gil-Monte et al., 2013). No entanto, este item não apresenta quaisquer problemas psicométricos em estudos anteriores usando a versão portuguesa do SBI, quer em estudos levados a cabo em Portugal (Figueiredo-Ferraz et al., 2013) ou no Brasil (Gil-Monte et al., 2010). Foi encontrada uma estrutura fatorial de quatro fatores que se ajusta ao modelo fatorial original, o que contribui para a validação psicométrica do modelo teórico de quatro fatores. A variância explicada pelo total das escalas, assim como a variância de cada uma das subescalas do questionário foi idêntica à de estudos anteriores onde foi realizada uma AFE com o SBI (Gil-Monte, 2008; Olivares \& Gil-Monte, 2007). 
Psicologia: Reflexão e Crítica, 27(2), 291-299.

Tabela 3

Matriz de Cargas Fatoriais para a Solução Utilizando Componentes Principais e Rotação Varimax (n=245)

\begin{tabular}{|c|c|c|c|c|}
\hline Item & Fator I & Fator II & Fator III & Fator IV \\
\hline Item 1 & 0,80 & $-0,05$ & $-0,07$ & $-0,04$ \\
\hline Item 5 & 0,82 & $-0,04$ & 0,03 & $-0,19$ \\
\hline Item 10 & 0,78 & $-0,11$ & $-0,12$ & $-0,06$ \\
\hline Item 15 & 0,85 & $-0,19$ & $-0,10$ & 0,02 \\
\hline Item 19 & 0,83 & $-0,11$ & $-0,10$ & $-0,03$ \\
\hline Item 8 & $-0,27$ & 0,11 & 0,27 & 0,70 \\
\hline Item 12 & 0,06 & 0,14 & 0,17 & 0,73 \\
\hline Item 17 & 0,10 & 0,05 & 0,23 & 0,73 \\
\hline Item 18 & $-0,44$ & 0,30 & 0,03 & 0,52 \\
\hline Item 2 & $-0,20$ & 0,19 & 0,62 & 0,26 \\
\hline Item 3 & 0,07 & -017 & 0,70 & 0,14 \\
\hline Item 6 & $-0,03$ & $-0,01$ & 0,72 & 0,18 \\
\hline Item 7 & $-0,06$ & 0,37 & 0,51 & 0,24 \\
\hline Item 11 & $-0,10$ & 0,26 & 0,70 & 0,05 \\
\hline Item 14 & $-0,27$ & 0,38 & 0,39 & $-0,27$ \\
\hline Item 4 & $-0,13$ & 0,68 & $-0,02$ & 0,14 \\
\hline Item 9 & $-0,37$ & 0,79 & 0,04 & $-0,04$ \\
\hline Item 13 & $-0,02$ & 0,74 & 0,15 & 0,18 \\
\hline Item 16 & $-0,01$ & 0,76 & 0,09 & 0,09 \\
\hline Item 20 & $-0,19$ & 0,01 & 0,48 & 0,42 \\
\hline Eigenvalue depois da rotação & 3,90 & 2,84 & 2,76 & 2,35 \\
\hline Percentagem da variância explicada & 19,51 & 14,19 & 13,79 & 11,76 \\
\hline
\end{tabular}

Nota. Valores $\geq|0,40|$ em negrito.

Tabela 4

Estatísticas Descritivas, Valores de Fiabilidade $\alpha$ de Cronbach e Correlações para as Variáveis do Estudo $(n=245)$

\begin{tabular}{lcccccccc}
\hline & $M(D P)$ & $\mathrm{As}$ & $\mathrm{Cu}$ & Intervalo & 1 & 2 & 3 & 4 \\
\hline 1. Entusiasmo pelo trabalho & $2,50(0,90)$ & $-0,38$ & $-0,41$ & $0-4$ & $(0,89)$ & & & \\
2. Desgaste psíquico & $1,51(0,79)$ & 0,41 & 0,24 & $0-4$ & $-0,30^{*}$ & $(0,74)$ & & \\
3. Indolência & $1,35(0,65)$ & 0,53 & 1,37 & $0-4$ & $-0,28^{*}$ & $0,44^{*}$ & $(0,73)$ & \\
4. Culpa & $1,01(0,65)$ & 0,56 & $-.0,11$ & $0-4$ & $-0,35^{*}$ & $0,42^{*}$ & $0,46^{*}$ & $(0,72)$ \\
\hline
\end{tabular}

Nota. Valores alfa de Cronbach aparecem na diagonal na matriz de correlações.

* $p<0,001$. 
Figueiredo-Ferraz, H., Gil-Monte, P. R., Queirós, C. \& Passos, F. (2014). Validação Factorial do "Spanish Burnout Inventory" em Policiais Portugueses.

Tanto os itens como as respectivas escalas do SBI apresentam valores de fiabilidade e validade aceitáveis, e os valores da homogeneidade corrigida para os itens estão muito acima de 0,30 , o que atesta a fiabilidade dos itens que compõem cada uma das escalas do questionário, sendo que o item 14 e o item 20 obtêm valores inferiores a 0,30 .

$\mathrm{O}$ valor para o alfa de Cronbach é superior a $0,70 \mathrm{em}$ todas em escalas, podendo afirmar-se que o instrumento apresenta suficiente consistência interna para avaliar a síndrome do burnout na população em estudo. Estes resultados são similares aos realizados em outros países com este instrumento noutros grupos profissionais, pelo que se pode afirmar que o instrumento apresenta validade transcultural e transocupacional (Gil-Monte, 2008; Olivares \& Gil-Monte, 2007).

A adaptação do SBI para português, na sua versão para policiais apresenta quatro dimensões idênticas às do modelo original: Entusiasmo pelo trabalho (5 itens), Desgaste psíquico (4 itens), Indolência (6 itens) e Culpa (5 itens).

Além disso, tanto os valores de assimetria como os valores de curtose obtidos neste estudo, quer para os itens, quer para as escalas do SBI, permitem afirmar que se ajustam a uma distribuição normal e que, mediante a sua aplicação, pode-se discriminar de maneira adequada os indivíduos que apresentam diferentes níveis dos sintomas avaliados.

O estudo apresenta algumas limitações, nomeadamente no que se refere às cargas fatoriais do item 20 que não se agrupa no fator que lhe corresponderia (Culpa). Esta limitação provavelmente se deve às características da amostra deste estudo e das especificidades das suas tarefas policiais, sendo necessários mais estudos com AFC.

Por outro lado, este estudo também apresenta relevância científica. Num estudo de meta-análise que tinha como objetivo sintetizar a consistência interna para as subescalas do MBI, Wheeler, Vassar, Worley e Barnes (2011) concluíram que as subescalas despersonalização e Realização pessoal no trabalho são problemáticas, dado que as estimativas médias de confiabilidade alcançadas foram baixas nas amostras utilizadas para o estudo, e assim advertem os investigadores e profissionais para os problemas existentes sobre a consistência entre os itens dessas subescalas. Nesse sentido, recomendam a realização de trabalho psicométrico adicional para refinar o conteúdo de alguns itens ou o uso de medidas alternativas de burnout com características psicométricas mais promissoras. Nesse sentido, a relevância deste estudo é que ele fornece evidências sobre as adequadas propriedades psicométricas de uma medida alternativa (SBI) para avaliar a síndrome do burnout numa amostra de policiais. Para poder seguir avançando na investigação sobre o burnout, é importante para os investigadores e profissionais terem um questionário com propriedades psicométricas aceitáveis e um conceito mais amplo do burnout do que a tradicional (Wheeler et al., 2011).

O SBI oferece também uma proposta teórica para explicar os diferentes tipos de burnout, tendo em consideração a incorporação da avaliação de culpa como um sintoma da síndrome, a fim de fazer uma identificação mais completa de indivíduos afetados por níveis críticos de burnout, e reconhecer sua influência sobre os transtornos de saúde (por exemplo, depressão) (Gil-Monte, 2008, 2012). Este é um contributo importante para a literatura, dado que oferece aos investigadores e profissionais um conceito mais abrangente da síndrome, o que pode facilitar o diagnóstico e tratamento de indivíduos com burnout, por exemplo, pela avaliação dos sentimentos de culpa e outros sintomas de burnout em intervenções destinadas a melhorar o apoio social (Gray-Stanley et al., 2010; Richardson \& Rothstein, 2008). Um diagnóstico nas fases iniciais do burnout pode prevenir o aumento da intensidade dos sintomas e ajudar numa mais rápida recuperação.

Como recomendação para estudos futuros, seria importante investigar se algumas variáveis sociodemográficas (p.e. genero) estabelecem diferenças na solução fatorial dada pelo instrumento. Como foi referido na anteriormente, a maioria dos estudos com o SBI foram realizados utilizando amostras de coletivos profissionais que na sua maioria eram formadas por mulheres. Da mesma forma, a profissão de policial possui características e valores culturais que podem originar diferenças nos valores psicométricos do instrumento, sendo por isso necessária a replicação deste estudos com amostras de policiais.

Como conclusão, pode-se afirmar que o SBI, na sua versão portuguesa para policiais, mesmo tendo algumas insuficiências, é um instrumento que obteve os requisitos suficientes de validade fatorial e consistência interna para avaliação da síndrome do burnout em policiais. Estes resultados podem ser úteis pois podem permitir avaliar a síndrome do burnout noutro grupo profissional distinto aos já estudados com este instrumento.

\section{Referências}

Abu-Hilal, M. M. (1995). Dimensionality of burnout: Testing for invariance across. Jordanian and Emirati teachers. Psychological Reports, 77, 1367-1375.

Afonso, J. M. P., \& Gomes, A. R. (2009). Stress ocupacional em profissionais de segurança pública: Um estudo com militares da Guarda Nacional Republicana. Psicologia: Reflexão e Crítica, 22, 294-303.

Agolla, J. E. (2009). Occupational stress among police officers: The case of Botswana Police Service. Research Journal of Business Management, 2, 25.

Akinola, M., \& Mendes, W. B. (2012). Stress-induced cortisol facilitates threat-related decision making among police officers. Behavioral Neuroscience, 126, 167-174.

Bhana, A., \& Haffejee, N. (1996). Relation among measures of burnout, job satisfaction, and role dynamics for a sample of South African child-care social workers. Psychological Reports, 79, 431-434.

Carlotto, M. S., \& Gonçalves, S. (2004). Factorial analysis of the Maslach Burnout Inventory (MBI) in a sample of teachers from private schools. Psicologia em Estudo, 9, 499-505.

Coleta, A., \& Coleta, N. (2008). Fatores de estresse ocupacional e coping entre policiais civis. Psico-USF, 13, 59-68. 
Durán, M. A., Montalbán, F. M., \& Stangeland, P. (2006). E síndrome de estar quemado en la policía: perfil de incidencia e influencia de factores socio-demográficos. Revista de Psicología Social, 21, 95-107.

Euwema, M. C., Kop, N., \& Bakker, A. B. (2004). The behaviour of police officers in conflict situations: How burnout and reduced dominance contribute to better outcomes. Work \& Stress, 18, 23-38.

Figueiredo-Ferraz, H., Gil-Monte, P. R., \& Grau-Alberola, E. (2013). Psychometric properties of the "Spanish Burnout Inventory" (SBI): Adaptation and validation in a Portuguesespeaking sample. European Review of Applied Psychology, 63, 33-40.

Freudenberger, H. J. (1974). Staff burn-out. Journal of Social Issues, 30, 159-165.

Gachter, M., Savage, D., \& Torgler, B. (2011). Gender variations of physiological and psychological strain amongst police officers. Gender Issues, 28, 66-93.

Gil-Monte, P. R. (2005). El sindrome de quemarse por el trabajo (Burnout): una enfermedad laboral en la sociedad del bienestar. Madrid, España: Psicología Pirámide.

Gil-Monte, P. R. (2008). Evaluación psicométrica del síndrome de quemarse por el trabajo (burnout): el cuestionario "CESQT”. In J. Garrido (Ed.), iMaldito trabajo! (pp. 269-291). Barcelona, España: Granica.

Gil-Monte, P. R. (2012). Influence of guilt on the relationship between burnout and depression. European Psychologist, 17, 231-236.

Gil-Monte, P. R., Carlotto, M. S., \& Gonçalves, S. (2010). Validação da versão brasileira do "Cuestionario para la Evaluación del Síndrome de Quemarse por el Trabajo" em professores. Revista de Saúde Pública, 44, 140-147.

Gil-Monte, P. R., Carretero, N., Roldán, M. D., \& Núñez-Román, E. (2005). Prevalencia del síndrome de quemarse por el trabajo (burnout) en monitores de taller para personas con discapacidad. Revista de Psicología del Trabajo y de las Organizaciones, 21, 107-123.

Gil-Monte, P. R., \& Figueiredo-Ferraz, H. (2013). Psychometric properties of the 'Spanish Burnout Inventory' among employees working with people with intellectual disability. Journal of Intellectual Disability Research, 57, 959-968. doi: 10.1111/j.1365-2788.2012.01591.x

Gil-Monte, P. R., Figueiredo-Ferraz, H., \& Valdez Bonilla, H. (2013). Factor analysis of the Spanish Burnout Inventory among Mexican prison employees. Canadian Journal of Behavioural Science, 45, 96-104. doi: 10.1037/a0027883.

Gil-Monte, P. R., \& Olivares, V. (2011). Psychometric properties of the "Spanish Burnout Inventory" in Chilean professionals working to physical disabled people. The Spanish Journal of Psychology, 14, 441-451.

Gil-Monte, P. R., Unda, S., \& Sandoval, J. I. (2009). Validez factorial del "Cuestionario para la Evaluación del Síndrome de Quemarse por el Trabajo" (CESQT) en una muestra de maestros mexicanos. Salud Mental, 32, 205-214.

Gold, Y., Bachelor, P., \& Michael, W. B. (1989). The dimensionality of a modified form of the Maslach Burnout Inventory for college students in a teacher-training program. Educational and Psychological Measurement, 49, 549-561.

Gray-Stanley, J. A., Muramatsu, N., Heller, T., Hughes, S., Johnson, T. P., \& Ramirez-Valles, J. (2010). Work stress and depression among direct support professionals: The role of work support and locus of control. Journal of Intellectual Disability Research, 54, 749-761.
Hackett, D. P., \& Violanti, J. M. (2003). Police suicide: Tactics for prevention. Springfield, IL: Charles C. Thomas.

Halbesleben, J. R. B., \& Demerouti, E. (2005). The construct validity of an alternative measure of burnout: Investigating the English translation of the Oldenburg Burnout Inventory. Work \& Stress, 19, 208-220.

Holland, P. J., \& Michael, W. B. (1993). The concurrent validity of the Holland Burnout Assessment Survey for a sample a middle school teachers. Educational and Psychological Measurement, 53, 1067-1077.

Iwanicki, E. F., \& Schwab, R. L. (1981). A cross validation study of the Maslach Burnout inventory. Educational and Psychological Measurement, 41, 1167-1174.

Johnson, S., Cooper, C., Cartwright, S., Donald, I., Taylor, P., \& Millet, C. (2005). The experience of work-related stress across occupations. Journal of Managerial Psychology, 20, 178-187.

Kalliath, T. J., O’Driscoll, M. P., Gillespie, D. F., \& Bluedorn, A. C. (2000). A test of the Maslach Burnout Inventory in three samples of healthcare professionals. Work \& Stress, 14, 35-50.

Kristensen, T. S., Borrritz, M., Villadsen, E., \& Christensen, K. B. (2005). The Copenhagen Burnout Inventory: A new tool for the assessment of burnout. Work \& Stress, 19, 192-207.

Mann-Feder, V., \& Savicki, V. (2003). Burnout in Anglophone and Francophone child and youth workers in Canada: A cross-cultural comparison. Child \& Youth Care Forum, 32, 337-354.

Marques-Pinto, A., Lima, M. L., \& Lopes da Silva, A. (2005). Fuentes de estrés, burnout y estrategias de coping en profesores portugueses. Revista de Psicología del Trabajo y de las Organizaciones, 21, 125-143.

Maslach, C., \& Jackson, S. E. (1981). Maslach Burnout Inventory Manual. Palo alto, CA: Consulting Psychologists Press.

Maslach, C., \& Jackson, S. E (1986). Maslach Burnout Inventory Manual ( $2^{\text {nd }}$ ed.) Palo Alto, CA: Consulting Psychologists Press.

Maslach, C., Jackson, S. E., \& Leiter, M. P. (1996). Maslach Burnout Inventory Manual ( $3^{\text {rd }}$ ed.). Palo Alto, CA: Consulting Psychologists Press.

Maslach, C., Jackson, S. E., \& Schwab, R. L. (1986). Maslach Burnout Inventory: Educators survey. Palo Alto, CA: Consulting Psychologists Press.

Maslach, C., Schaufeli, W. B., \& Leiter, M. P. (2001). Job burnout. Annual Review Psychology, 52, 397-422.

Mercado, A., \& Gil-Monte, P. R. (2012). Características psicométricas del "Cuestionario para la evaluación del Síndrome de quemarse por el trabajo" en maestros mexicanos. Revista de Educación, 359, 260-273. doi: 10-4438/1988-592X-RE2010-359-094.

Mota-Cardoso, R., Araújo, A., Carreira Ramos, R., Gonçalves, G., \& Ramos, M. (2002). Ostress nos professores portugueses - Estudo IPSSO 2000. Porto, Portugal: Porto.

Nunnally, N. C. (1978). Psychometric theory. New York: McGraw-Hill.

Olivares, V. E., \& Gil-Monte, P. R. (2007). Análisis de las propiedades psicométricas del "Cuestionario para la Evaluación del Síndrome de Quemarse por el Trabajo” (CESQT) en profesionales chilenos. Ansiedad y Estrés, 13, 229-240.

Pancheri, P., Marini, A., Tarsitani, L., Rosati, V., Biondi, M., \& Tomei, F. (2002). Assessment of subjective stress in the municipal police force of the city of Rome. Stress and Health, $18,127-132$. 
Figueiredo-Ferraz, H., Gil-Monte, P. R., Queirós, C. \& Passos, F. (2014). Validação Factorial do “Spanish Burnout Inventory" em Policiais Portugueses.

Pedrabissi, L., Santinello, M., \& Vialetto, A. (1994). La sindrome del burnout: una ricercatra gli insegnanti e gli operatori dei servizi psichiatrici del Friuli-Venezia Giulia. Pordedone, Italia: Biblioteca dell'Immagine.

Peeters, M. A. G., \& Rutte, C. G. (2005). Time-management behavior as a moderator for the job demand-control interaction. Journal of Occupational Health Psychology, 10, 64-75.

Pines, A. M., Aronson, E., \& Kafry, D. (1981). Burnout: From tedium to personal growth. In C. Cherniss (Ed.), Staff burnout: Jon stress in the human services. New York: Free Press.

Pines, A. M., \& Keinan, G. (2005). Stress and burnout: The significant difference. Personality and Individual Differences, 39, 625-635.

Pines, A. M., \& Keinan, G. (2006). Stress and burnout in Israeli border police. International Journal of Stress Management , 13, 519-540.

Purvanova, R. K., \& Muros, J. P. (2010). Gender differences in burnout: A meta-analysis. Journal of Vocational Behavior, 77, 168-185.

Ransley, J., \& Mazerolle, L. (2009). Policing in an era of uncertainty. Police Practice and Research, 10, 365-381.

Ranta, R. S., \& Sud, A. (2008). Management of stress and burnout of police personnel. Journal of the Indian Academy of Applied Psychology, 34, 29-39.

Ravelo, R. M., Garcia, L. A., \& Dorta, A. V. (2009). Ránking de estresores en la policía local de Canárias. Anuario de Psicología Jurídica, 18, 73-79.

Richardson, K. M., \& Rothstein, H. R. (2008). Effects of occupational stress management intervention programs: A meta-analysis. Journal of Occupational Health Psychology, 13, 69-93.

Sá, L., \& Fleming, M. (2008). Bullying, burnout, and mental health among Portuguese nurses. Issues in Mental Health Nursing, 29, 411-426.

Schaible, L. M., \& Gecas, V. (2010). The impact of emotional labor and value dissonance on burnout among police officers. Police Quarterly, 13, 316-341.

Soderfeldt, M., Soderfeldt, B., Warg, L. E., \& Ohlson, C. G. (1996). The factor structure of the Maslach Burnout Inventory in two Swedish human service organizations. Scandinavian Journal of Psychology, 37, 437-443.

van Gelderen, B. R., Bakker, A. B., Konijn, E. A., \& Demerouti, E. (2011). Daily suppression of discrete emotions during the work of police service workers and criminal investigation officers. Anxiety, Stress \& Coping, 24, 515-537.

Violanti, J., Burchfiel, C., Hartley, T., Mnatsakanova, A., Fekedulegn, D., Andrew, M., ... Vila, B. (2009). Atypical work hours and metabolic syndrome among police officers. Archives of Environmental \& Occupational Health, 64, 194-201.

Wheeler, D. L., Vassar, M., Worley, J. A., \& Barnes, L. L. B. (2011). A reliability generalization meta-analysis of coefficient alpha for the Maslach Burnout Inventory. Educational and Psychologica Measurement, 71, 231-244. 